\title{
Abundances and radial velocity analysis of BW Vulpeculae ${ }^{\star}$
}

\author{
A. Stankov ${ }^{1}$, I. Ilyin ${ }^{2}$, and C. V. M. Fridlund ${ }^{1}$ \\ 1 SCI-SA, Research and Scientific Support Department of ESA, ESTEC, 2200 AG Noordwijk, The Netherlands \\ e-mail: Malcolm.Fridlund@esa.int \\ 2 Astronomy Division, PO Box 3000, 90014 University of Oulu, Finland \\ e-mail: ilya.ilyin@oulu.fi
}

Received 12 March 2003 / Accepted 10 June 2003

\begin{abstract}
We present the results of the analysis of 56 échelle spectra of the $\beta$ Cephei star BW Vulpeculae. The data were obtained during three nights with the SOFIN spectrograph at the Nordic Optical Telescope and cover a wavelength range from $3650 \AA$ to $7340 \AA$. We compare the observed spectrum with a synthetic spectrum calculated from a model atmosphere for this star utilizing the program ATLAS9 by Kurucz. From this, we determine a projected rotational velocity, $v \sin i$, of $24 \mathrm{~km} \mathrm{~s}^{-1}$.

Detailed spectral line identification and an abundance analysis for this star are performed. From these analyses we find that $\mathrm{Ne}$, $\mathrm{Si}, \mathrm{He}$, and Ar are over-abundant in BW Vulpeculae, while the abundances for N, S, O, Fe, C, and Al are solar.

Finally, a comparison of radial velocity curves from six different layers of BW Vulpeculae's atmosphere is presented, corresponding to the formation depths of different elements. We show that at least six of these layers move independently from each other.
\end{abstract}

Key words. stars: abundances - stars: individual: BW Vulpeculae - stars: oscillations - stars: variables: Cepheids

\section{Introduction}

BW Vulpeculae (=HD 199140, $V=6.54 \mathrm{mag}$ ) is a short period variable star of the $\beta$ Cephei type. The pulsational instability in these stars is driven by the $\kappa$-mechanism operating in the zone of the bump in metal opacity. The opacity of metals has been underestimated for many years and only after the recalculation of the opacities by the OPAL group (Iglesias \& Rogers 1991) and their availability for the astrophysical community, pulsational stability studies of $\beta$ Cephei models have shown conclusively that these new opacities are indeed driving pulsation in $\beta$ Cephei stars (e.g. Moskalik \& Dziembowski 1992; Dziembowski \& Pamyatnykh 1993).

This mechanism is based on the theory that there exist ionization zones ( $\mathrm{H}, \mathrm{He}$ II and metals) in pulsating stars, which are all in different optical depths, where radiative flux coming from the interior of the star can partly be blocked during the phase of compression and be converted into kinetic energy. Photon energy is transformed into mechanical movement and this excess energy can then be used for the pulsation.

BW Vul is mono-periodic with a $4.825 \mathrm{~h}$ brightness variation, increasing by $2.34 \mathrm{~s} /$ century (Pigulski 1993).

Send offprint requests to: A. Stankov, e-mail: Anamarija.Stankov@esa.int

* Based on observations made with the Nordic Optical Telescope, operated on the island of La Palma jointly by Denmark, Finland, Iceland, Norway, and Sweden, in the Spanish Observatorio del Roque de los Muchachos of the Instituto de Astrofisica de Canarias.
The pulsation period of $P=0.20104444 \mathrm{~d}$ has been well determined (Sterken et al. 1993). Recent studies of its pulsational behavior, applying the moment method (Aerts et al. 1992) and the method of photometric amplitudes (e.g. Heynderickx et al. 1994), show that it pulsates in the fundamental radial mode with up to 8 harmonics (Aerts et al. 1995).

BW Vul is a special case among the $\beta$ Cephei stars, exhibiting many atypical properties. Usually, the $\kappa$-mechanism leads in $\beta$ Cephei stars to small amplitudes of $\sim 0.02 \mathrm{mag}$. However, BW Vul has the largest lightamplitude among the known $\beta$ Cephei stars, with an amplitude of $\sim 0.2$ mag peak to peak in the visual and $\sim 1.2$ mag in the ultraviolet.

In addition, although the $\kappa$-mechanism usually results in line profile variations, there is clear line doubling in the spectra of BW Vul, caused by strong non-linear behavior. One possible explanation for this phenomenon is the propagation of shock waves through the atmosphere of the star (Mathias et al. 1998). This process is described further in Sect. 5.

Finally, BW Vul exhibits extensive radial velocity variations of $\sim 200 \mathrm{~km} \mathrm{~s}^{-1}$. The velocity curve is unusually complex, marked by a still stand phase with nearly constant radial velocity. Mathias et al. (1998) discovered a fine structure within this so-called still stand, produced by an inward shock front bouncing off a deep layer of the photosphere and a slight outwards motion of the shock wave.

To date, no satisfactory explanation has been provided for much of BW Vul's atypical behavior. In this paper we aim to 
Table 1. Spectroscopic range of each order of the échelle spectra with resolution $R=70000$.

\begin{tabular}{cc||cc}
\hline \hline Order & Spectral Range $[\AA]$ & Order & Spectral Range $[\AA]$ \\
\hline 34 & $6542-6592$ & 46 & $4850-4886$ \\
35 & $6358-6440$ & 48 & $4648-4683$ \\
38 & $5854-5898$ & 49 & $4553-4587$ \\
39 & $5704-5746$ & 50 & $4462-4496$ \\
41 & $5248-5466$ & 51 & $4374-4407$ \\
43 & $5172-5228$ & 52 & $4290-4323$ \\
\hline
\end{tabular}

complete a detailed abundance analysis of this star, and to study radial velocities of different elements, and hence different layers of the atmosphere. The results of these investigations will provide significant grounding for further research on this unusual star.

The observations are described in Sect. 2. We derive a synthetic spectrum for the first time for this star and this process is outlined in Sect. 3. In Sect. 4, we show the results of the abundance analysis and in Sect. 5 we discuss line doubling effects. In Sect. 6 the results of the calculation of the projected rotational, systemic and radial velocities are presented. Section 7 summarizes the results of our study. Finally, in Sect. 8 we outline our future work on BW Vul.

\section{Observations}

The spectra were obtained with the high-resolution échelle spectrograph SOFIN (Tuominen et al. 1999) mounted at the Cassegrain focus of the $2.56 \mathrm{~m}$ Nordic Optical Telescope (NOT) in La Palma, Spain. These observations of BW Vul were among the first made with the NOT. The medium resolution camera (camera $2, R \approx 70000$ ) was used for the first data set of 40 individual exposures of 13 orders of about $50 \AA$ each and they yielded a typical signal/noise of about 200 . In this medium resolution mode 6 orders in 23 phases containing $\mathrm{H} \alpha(\lambda 6562.797 \AA)$ and 7 orders in 17 phases containing $\mathrm{H} \beta(\lambda 4861.323 \AA$ ) were obtained. The spectroscopic range of each order of these échelle spectra are presented in Table 1. A second set of data of lower resolution $(R \approx 30000)$ was observed with camera 3 . Here there were 16 exposures of 31 orders obtained. The spectroscopic range of each order of the low resolution data can be seen in Table 2 . These data sets together resulted in a total of 56 échelle spectra for BW Vul which were used for this study.

The reduction was completed using the échelle spectrum reduction package 4A (Ilyin 2000) including bias subtraction, flat fielding, scattered light subtraction, weighted spectrum extraction and wavelength calibration with the use of a ThAr spectrum. Telluric lines were used to correct the wavelength positions of the spectra caused by the bending of the spectrograph, temperature distortions, and the slit effect of the seeing image.

The medium resolution observations were carried out in 1993 (June 30th and July 4th). The low resolution data were obtained on August 29th, 1994.
Table 2. Spectroscopic range of each order of the échelle spectra with resolution $R=30000$.

\begin{tabular}{|c|c|c|c|}
\hline Order & Spectral Range $[\AA]$ & Order & Spectral Range $[\AA]$ \\
\hline 31 & $7190-7340$ & 46 & $4740-4845$ \\
\hline 32 & $6960-7120$ & 49 & $4655-4560$ \\
\hline 33 & $6750-6900$ & 47 & $4640-4745$ \\
\hline 34 & $6550-6700$ & 48 & $4545-4645$ \\
\hline 35 & $6360-6510$ & 50 & $4365-4465$ \\
\hline 36 & $6180-6325$ & 51 & $4280-4380$ \\
\hline 37 & $6020-6160$ & 52 & $4200-4295$ \\
\hline 38 & $5860-5995$ & 53 & $4125-4215$ \\
\hline 39 & $5710-5840$ & 54 & $4050-4140$ \\
\hline 40 & $5570-5695$ & 55 & $3980-4065$ \\
\hline 41 & $5430-5560$ & 56 & 3910-3995 \\
\hline 42 & $5300-5420$ & 57 & $3840-3930$ \\
\hline 43 & $5180-5295$ & 58 & $3775-3860$ \\
\hline 44 & $5060-5190$ & 59 & $3710-3795$ \\
\hline 45 & $4950-5060$ & 60 & $3650-3730$ \\
\hline 45 & $4840-4950$ & & \\
\hline
\end{tabular}

\section{Synthetic spectrum for BW Vul}

In order to perform an abundance analysis, it is first necessary to synthesize a spectrum for BW Vul. The spectroscopic data set obtained for these studies covers an extensive wavelength range from $3650 \AA$ to $7340 \AA$. This range provides a good basis for line identification and abundance analysis as well as for determination of the projected rotational, systemic and radial velocities in BW Vul.

To produce a synthetic spectrum, first $T_{\text {eff }}$ and $\log g$ for this star need to be determined. These parameters are then used for the calculation of a model atmosphere from which the spectrum is synthesized. Details of the procedure are provided below.

- Calibration: The effective temperature $\left(T_{\text {eff }}\right)$ and $\log g$ are obtained from calibration of Strömgren photometric indices (Hauck \& Mermilliod 1998) using the program templogg (Kupka \& Lueftinger 2001). templogg is a tool for the determination of fundamental stellar parameters from photometric observations. It is a compilation of a large sample of calibrations for the Strömgren- $\beta$ and the Geneva photometric systems. An automatic selection of the most suitable transformation between observed colors and stellar parameters allows for the determination of $T_{\text {eff }}, \log g$ and metallicity from observed colors. The use of templogg results for BW Vul in $T_{\text {eff }}=24432 \mathrm{~K}$ and $\log g=3.73$.

We verified the results of this program using polynomial functions to calculate $T_{\text {eff }}$ and $\log g$ from Strömgren color indices. For B-type stars, Balona (1984) obtained these polynomial functions by calibrating effective temperatures and bolometric corrections in terms of Strömgren $c_{0}$ and $\beta$ indices. The $c_{0}$ index $\left(c_{0}=c_{1}-0.19 E(b-y)\right)$ provides a precise measurement of the Balmer discontinuity and the $\beta$ index measures the strength of $\mathrm{H} \alpha$. Using these polynomial functions, we obtain $T_{\text {eff }}=23850 \pm 940$ and 


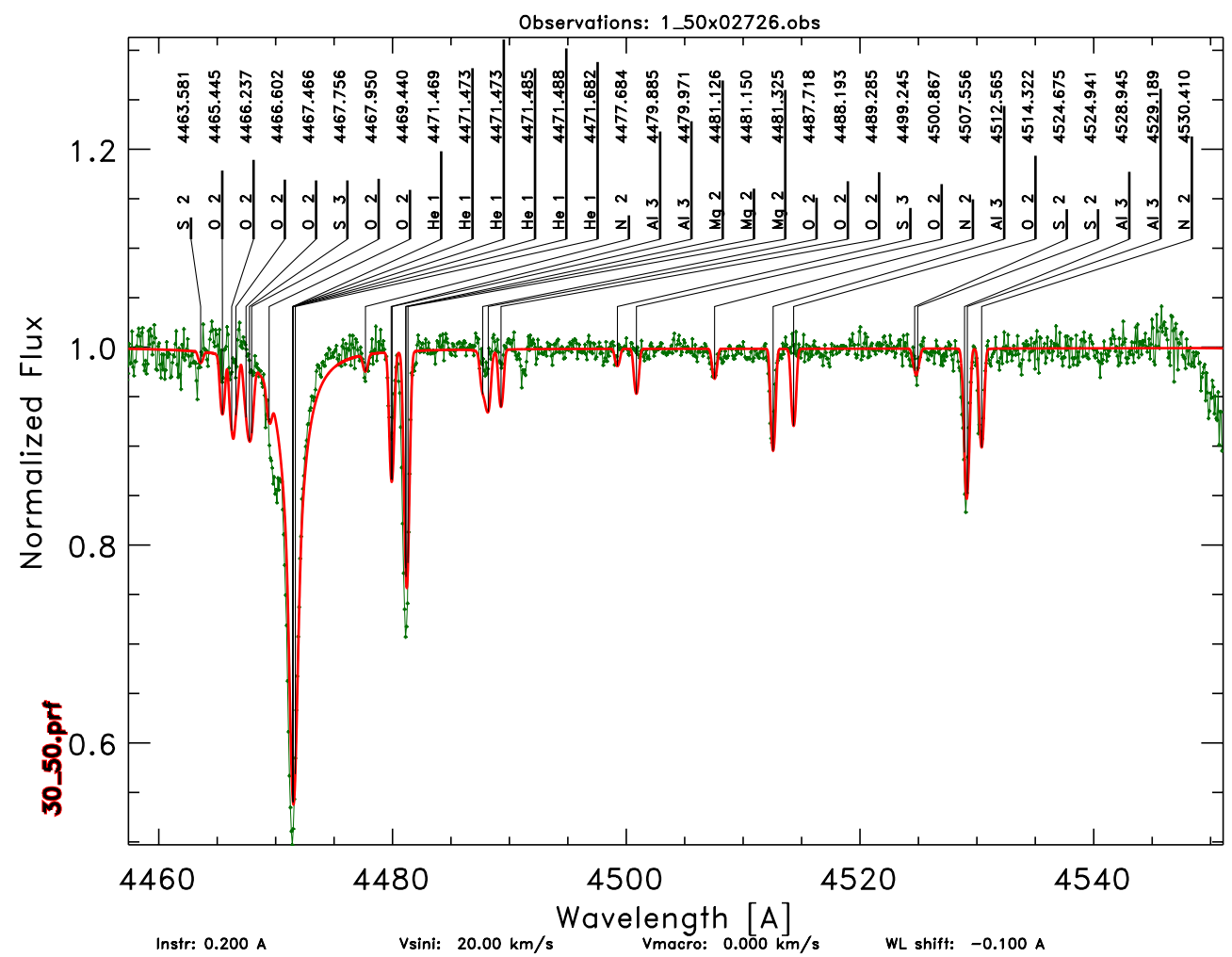

Fig. 1. Comparison of observed and synthesized spectrum of BW Vulpeculae for the spectral region between $4460 \AA$ and $4550 \AA$. The green line represents the observed spectrum and the red line represents the synthesized spectrum.

$\log g=3.76 \pm 0.04$. Nota bene, both calculations provide the same values for $T_{\text {eff }}$ and $\log g$ and they agree very well with previously determined values (e.g. $T_{\text {eff }}=23227 \mathrm{~K}$ by Aerts et al. 1995).

- Model atmosphere: The standard ATLAS9 code by Kurucz (1992) was used to generate the model atmosphere. The input parameters were $T_{\text {eff }}$ and $\log \mathrm{g}$, as calculated from the previous step, metallicity $(Z=0.02)$, an estimated value for the micro turbulence of $v_{\text {micro }}=2 \mathrm{~km} \mathrm{~s}^{-2}$, and an estimated starting value for the projected rotational velocity $v \sin i$ of $12 \mathrm{~km} \mathrm{~s}^{-2}$.

- Synthesis: The synthetic spectrum was generated from the model atmosphere using the synth program (Piskunov 1992). The metallicity was taken from Pamyatnykh (1999). The atomic line parameters were extracted from the VALD ${ }^{1}$ database (Kupka et al. 1999; Ryabchikova et al. 1998; Piskunov et al. 1995). VALD is regularly updated with critically evaluated data sets. The program synth solves the radiative transport equations for every single wavelength point using an adaptive grid.

The synthesized spectrum of BW Vul which was obtained using the procedures described above closely matches the observed spectrum. In Figs. 1 and 2 we illustrate how close the synthesized spectrum resembles the observed spectrum. Figure 1 shows the observed and the synthesized spectrum of BW Vul in the wavelength range between $4460 \AA$ and $4550 \AA$.

\footnotetext{
${ }^{1}$ http://www.astro. univie.ac.at/ vald/
}

This part of the spectrum contains the N II line at $4507.6 \AA$ and the Al III line at $4512.6 \AA$. Figure 2 shows the observed and the synthesized spectrum of BW Vul in the wavelength range between $4740 \AA$ and $4840 \AA$. This part of the spectrum contains two strong Si III triplets (at $4819 \AA$ and $4829 \AA$ ) and the strong Si III line at $4813.3 \AA$. Here we also find the $\mathrm{S}$ II line at 4815.6 $\AA$ and two N II lines (at $4779.7 \AA$ and $4788.1 \AA$ ). All lines in the observed spectrum can also be identified in the synthesized spectrum, and there are no superfluous lines in the latter.

Therefore, the calculated model atmosphere must closely resemble the observed atmosphere of BW Vul, and the synthesized spectrum enables us to perform the following analyses with confidence.

\section{Abundance analysis}

The synthetic spectrum produced for BW Vul is used to complete the first ever abundance analysis for this star. Compositional anomalies within this star may help to explain some of its atypical behavior.

The first step in the abundance analysis is to compare the observed and the calculated spectra using the program rotate $^{2}$. This program allows one to manually adjust the radial velocity, rotational velocity, instrumental profile, and macro turbulence, and to measure equivalent widths. From this, all lines in the present data set of BW Vul are identified. In

\footnotetext{
2 Rotate: Prof. Nikolai Piskunov, Dept. of Astronomy and Space Physics, Uppsala University, Box 515S-751 20 Uppsala, Sweden.
} 


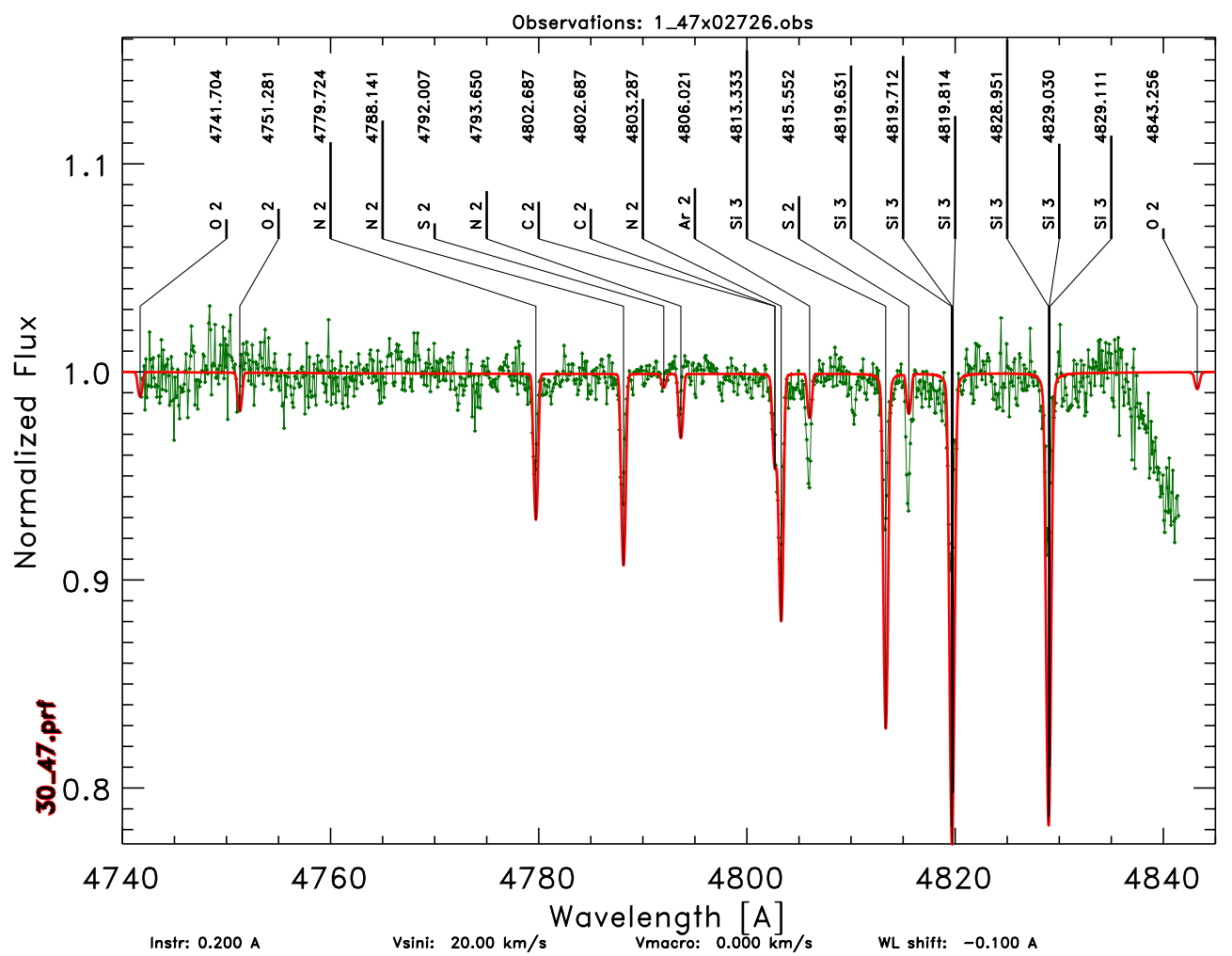

Fig. 2. Comparison of observed and synthesized spectrum of BW Vulpeculae for the spectral region between $4740 \AA$ and $4840 \AA$. The green line represents the observed spectrum and the red line represents the synthesized spectrum.

Table 3, we give a line list together with the ionization levels of the elements and their laboratory wavelengths of the medium resolution data.

In order to determine the abundance of each element identified in this star, abundances of the synthetic spectra are adjusted iteratively until a match is found between the equivalent widths of the lines in the observed and the synthetic spectrum. Solar values are adopted as a starting point for this iterative process. Each element is studied using as many unblended lines as are available. We would like to stress that for elements which exhibit lines not as strong as for $\mathrm{Si}$ or $\mathrm{He}$, we used only those lines which have very good signal to noise ratios and can therefore be used for accurate abundande determination.

Using this procedure, the atmospheric abundances of 10 elements in BW Vul could be determined. The results of the abundance analysis are presented in Table 4, listing the solar abundances $\odot$, the abundances determined for BW Vul, the difference $\Delta$ between these values, and the number of lines used for the analysis to determine the abundances. This last number allows for quantification of the given errors. It is difficult to obtain accurate abundances for this star because the line profiles change considerably within each pulsation cycle. Nevertheless, these abundance measurements are unprecedented in the literature and are of considerable value for the interpretation of the peculiar behavior of this star.

In Fig. 3 we present the abundance determination for nitrogen for which there were the most lines (18) available. The abundance of $-3.75 \pm 0.27$ dex indicates solar abundance for nitrogen. The elemental abundances in comparison to solar

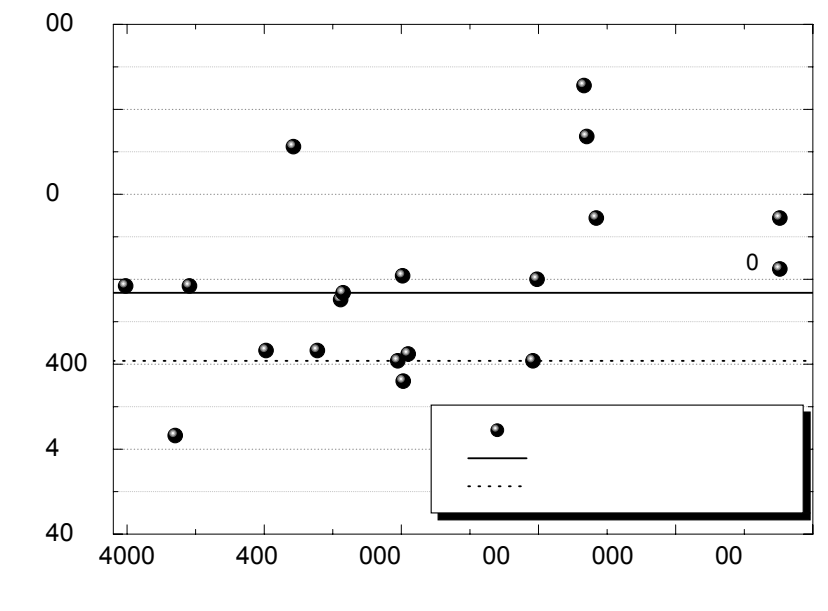

Fig. 3. Nitrogen abundance as determined from 18 lines. The circles mark the abundances obtained from the different nitrogen lines. The numbers next to them denote the number of single measurements of different phases in one order which were averaged, representing the phases per order usable for calculation. The full line marks the average abundance and the dashed line marks the solar abundance. Note that the error on the abundance for BW Vul is 0.27 dex.

values are as follows:

- over-abundant elements: Ne, Si, Ar, and He;

- elements with solar abundance: N, S, O, Fe, C, and Al

Note that the abundance for Ar is determined from a single spectral line. The error is estimated from the standard deviation of the mean value of the abundance obtained from all phases in one order. 
Table 3. Table of all identified lines in the medium resolution spectra from BW Vul. Groups of elements without line spacing mark blends. Ion stands for element and $\lambda[\AA]$ is the central wavelength.

\begin{tabular}{|c|c|c|c|c|c|}
\hline Ion & $\lambda[\AA]$ & Ion & $\lambda[\AA]$ & Ion & $\lambda[\AA]$ \\
\hline \multirow[t]{2}{*}{$\mathrm{C}_{\text {II }}$} & 6582.882 & $\mathrm{~S}_{\text {II }}$ & 5428.655 & $\mathrm{Al}$ III & 4480.009 \\
\hline & & & & Al III & 4479.971 \\
\hline \multirow[t]{2}{*}{ C II } & 6578.053 & S II & 5207.038 & $\mathrm{Al}$ III & 4479.885 \\
\hline & & O II & 5206.650 & & \\
\hline \multirow[t]{2}{*}{ H I } & 6562.797 & O II & 5206.715 & $\mathrm{He} \mathrm{I}$ & 4471.682 \\
\hline & & & & $\mathrm{Ne} I$ & 4471.587 \\
\hline \multirow[t]{2}{*}{$\mathrm{Ne} I$} & 6402.246 & Fe III & 5194.160 & He I & 4471.488 \\
\hline & & $\mathrm{Fe}$ III & 5193.909 & He I & 4471.485 \\
\hline \multirow[t]{2}{*}{$\mathrm{Ne} I$} & 6382.991 & & & He I & 4471.473 \\
\hline & & O II & 5190.565 & He I & 4471.469 \\
\hline \multirow[t]{2}{*}{ N II } & 6379.616 & O II & 5190.494 & & \\
\hline & & $\mathrm{N}$ II & 5190.375 & O II & 4395.935 \\
\hline \multirow[t]{2}{*}{ Si II } & 6371.371 & & & Fe III & 4395.755 \\
\hline & & N II & 5179.347 & & \\
\hline Fe III & 5876.337 & & & K II & 4388.159 \\
\hline He I & 5875.966 & O II & 5175.986 & C III & 4388.017 \\
\hline He I & 5875.640 & & & He I & 4387.929 \\
\hline $\mathrm{He} \mathrm{I}$ & 5875.625 & O II & 5175.908 & & \\
\hline He I & 5875.615 & & & O II & 4319.865 \\
\hline He I & 5875.614 & N II & 5175.888 & Fe II & 4319.680 \\
\hline \multirow[t]{2}{*}{$\mathrm{He} \mathrm{I}$} & 5875.599 & & & O II & 4319.625 \\
\hline & & H I & 4861.323 & & \\
\hline Fe III & 5891.904 & Si II & 4861.074 & C II & 4317.266 \\
\hline \multirow[t]{2}{*}{ C II } & 5891.597 & O II & 4860.962 & O II & 4317.136 \\
\hline & & $\mathrm{Fe}$ II & 4860.677 & & \\
\hline \multirow[t]{2}{*}{ Si III } & 5739.734 & N II & 4860.162 & Fe III & 4310.355 \\
\hline & & & & Fe III & 4310.337 \\
\hline Al III & 5722.730 & O II & 4676.231 & & \\
\hline Fe III & 5722.468 & & & K II & 4309.096 \\
\hline \multirow[t]{2}{*}{ Fe III } & 5722.023 & O II & 4673.732 & O II & 4309.077 \\
\hline & & Si II & 4673.284 & O II & 4308.891 \\
\hline \multirow[t]{2}{*}{ Si III } & 5716.287 & Si II & 4673.256 & & \\
\hline & & & & O II & 4304.166 \\
\hline \multirow[t]{2}{*}{ N II } & 5710.766 & O II & 4661.635 & O II & 4303.833 \\
\hline & & & & O II & 4303.615 \\
\hline \multirow[t]{2}{*}{ Si III } & 5704.590 & O II & 4650.838 & Fe II & 4303.176 \\
\hline & & & & O II & 4303.124 \\
\hline N II & 5454.214 & Ar II & 4579.349 & O II & 4302.901 \\
\hline \multirow[t]{2}{*}{ S II } & 5453.885 & O II & 4579.269 & & \\
\hline & & & & $\mathrm{Ne}$ II & 4297.017 \\
\hline N II & 5452.075 & Si III & 4574.757 & Fe III & 4296.851 \\
\hline Si III & 5451.960 & & & $\mathrm{Fe}$ III & 4296.814 \\
\hline Ni III & 5451.874 & Si III & 4567.840 & Ti III & 4296.693 \\
\hline \multirow[t]{2}{*}{ Si III } & 5451.455 & & & & \\
\hline & & Mg II & 4481.325 & O II & 4294.871 \\
\hline \multirow[t]{2}{*}{ S II } & 5432.797 & Mg II & 4481.150 & S II & 4294.402 \\
\hline & & Mg II & 4481.126 & O II & 4294.037 \\
\hline
\end{tabular}

No abundance for $\mathrm{Mg}$ could be determined because the Mg III triplet at $4481 \AA$ is blended with the Al III doublet at $4479 \AA$ as can be seen in Fig. 1 .
Table 4. Results of the abundance analysis.

\begin{tabular}{l|c|c|c|c}
\hline \hline Element & $\begin{array}{c}\odot \\
{[\text { dex }]}\end{array}$ & $\begin{array}{c}\text { BW Vul } \\
{[\text { dex }]}\end{array}$ & $\Delta$ & \# of lines \\
\hline $\mathrm{Ne}$ & -3.95 & -2.75 & $1.20 \pm 0.07$ & 2 \\
$\mathrm{Si}$ & -4.49 & -3.49 & $1.00 \pm 0.48$ & 7 \\
$\mathrm{Ar}$ & -5.48 & -4.85 & $0.63 \pm 0.06$ & 1 \\
$\mathrm{He}$ & -1.05 & -0.73 & $0.32 \pm 0.02$ & 2 \\
\hline $\mathrm{N}$ & -3.99 & -3.75 & $0.24 \pm 0.27$ & 18 \\
$\mathrm{~S}$ & -4.82 & -4.63 & $0.19 \pm 0.33$ & 4 \\
$\mathrm{O}$ & -3.11 & -3.16 & $-0.05 \pm 0.25$ & 9 \\
$\mathrm{Fe}$ & -4.37 & -4.42 & $-0.05 \pm 0.14$ & 5 \\
$\mathrm{C}$ & -3.48 & -3.59 & $-0.11 \pm 0.13$ & 4 \\
$\mathrm{Al}$ & -5.57 & -4.72 & $0.85 \pm 1.36$ & 2 \\
\hline
\end{tabular}

The abundance analysis highlights an exciting possibility concerning BW Vul. There is a large $\Delta=0.32 \pm 0.02$ dex overabundance of helium (obtained from two strong and well determined He lines at $\lambda 4713 \AA$ and $\lambda 4437 \AA$ ) which may be due to the presence of a strong magnetic field diminishing the mixing of elements. A stronger magnetic field could also be related to the shock phenomena occurring in this star. There were four He lines available in total for study, two of which were blended with other elements and therefore discarded. The abundance determined from the remaining two lines has an extremely low error and can therefore be used with confidence.

Using the present data alone, it is not possible to determine whether or not BW Vul has a strong magnetic field. Future work will address this issue through observing campaigns to measure the circular polarization signal of BW Vul and determine whether such a magnetic field is present.

\section{Line doubling}

Line doubling is the formation of a red and a blue component for a spectral line, often occurring either as an effect of nonradial pulsation or due to shock waves.

In the case of BW Vul, the line doubling is best seen in Fig. 4 (oval marks) for $\mathrm{H} \alpha$ at phase $\Phi=0.93$ and $\Phi=0.13$ and for Si III at $\Phi=0.87$ and $\Phi=0.93$.

It is unlikely that line doubling in BW Vul is caused by non-radial modes as Aerts et al. (1995) demonstrated that this star pulsates in a radial mode. Therefore, it is far more likely that the line doubling originates from shock waves as described by Mathias et al. (1998). The shock front that moves outwards (between $\Phi=0.96$ and 0.52 ) is induced by the $\kappa$-mechanism, resulting in the first line doubling. The second line doubling (between $\Phi=0.59$ and 0.93 ) is most probably caused by the formation of a shock wave during the in-falling motion of the atmosphere. This means that the discontinuities occur before and after the still stand phase and they follow phases of rapid velocity changes.

As a consequence of this line doubling phenomenon it is difficult to measure spectral line centers. One can either determine the center of both the red and blue line components, or use the whole profile to obtain the line center. As we do not intend to use the information provided by the blue and red 

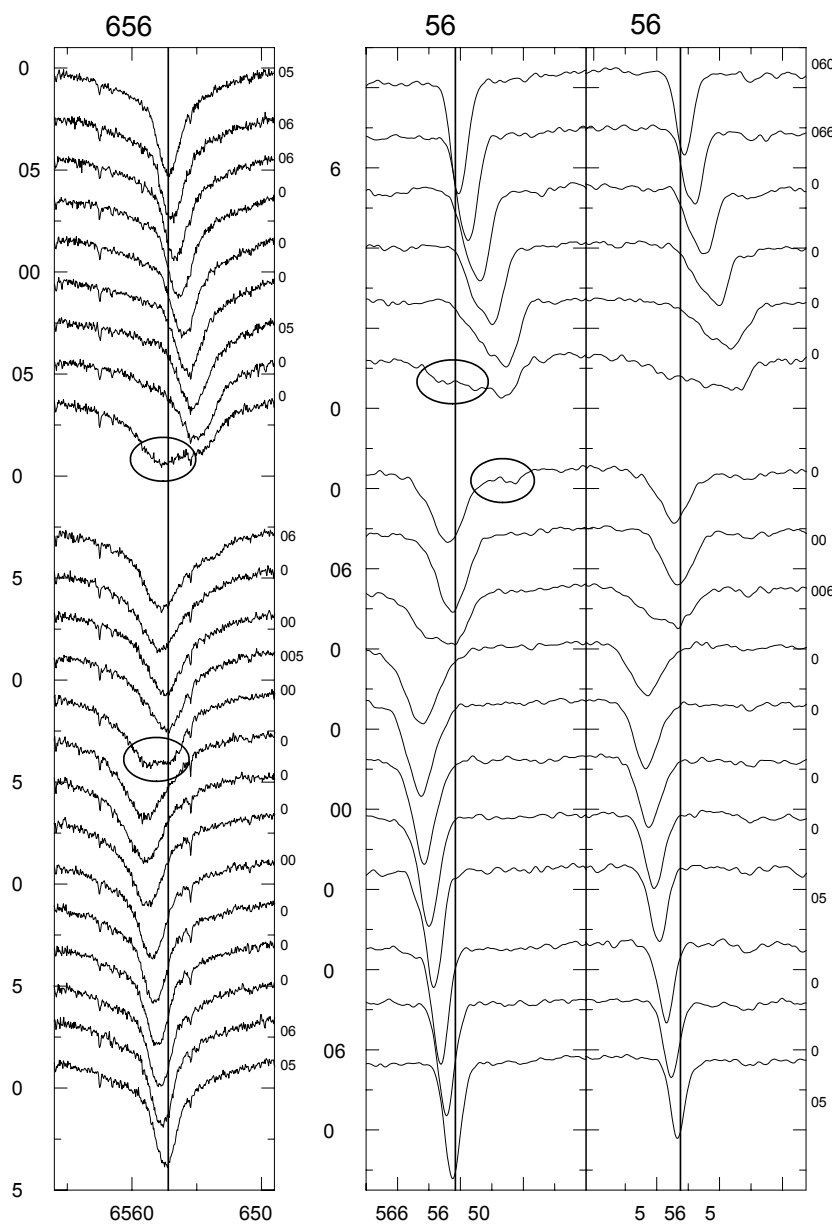

Fig. 4. Line doubling: spectra of the $\mathrm{H} \alpha$ line at $6562.797 \AA$, the Si III line at $4567.840 \AA$ and the Si III line at $4574.757 \AA$. The pulsation phase $\phi$ is given on the right of each spectrum. The left panel shows the $\mathrm{H} \alpha$ line, the right panel shows the two Si III lines. The vertical line represents the laboratory wavelength; ellipses mark visible line doubling.

components in this study, the central wavelength is measured from the whole profile.

\section{Determination of velocities}

The projected rotational, systemic and radial velocities for BW Vul are measured in order to produce a more comprehensive physical model for the star and to measure the differential motion of layers within its atmosphere.

\subsection{Projected rotational velocity $v \sin i$}

The determination of the projected rotational velocity $v \sin i$ allows for quantification of line broadening caused by stellar rotation, and its differentiation from other effects such as thermal or pressure broadening.

For BW Vul, the broad wavelength range of the data set $(3690 \AA)$ allows for the derivation of the mean $v \sin i$ from 16 different spectral lines. For each of the 16 lines, the $v \sin i$ of the star is adjusted until a match is found between the observed

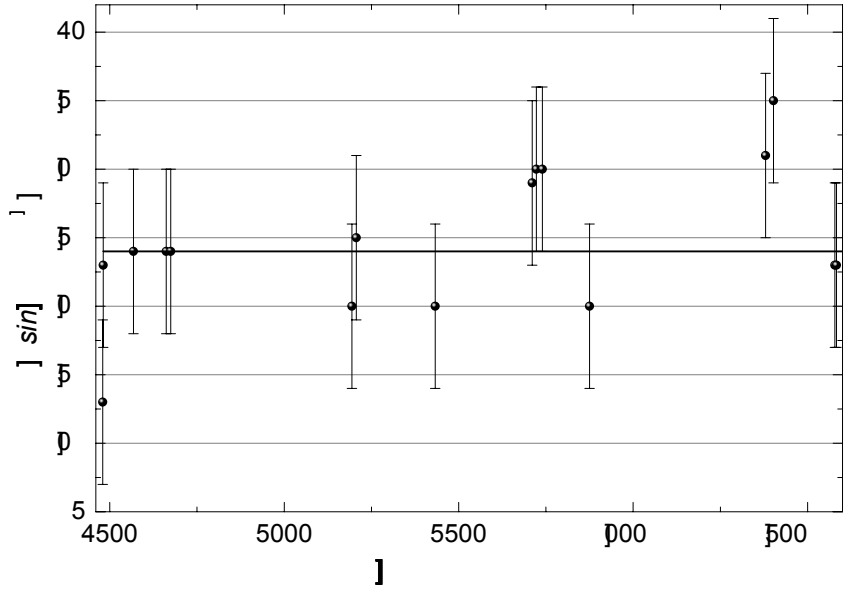

Fig. 5. $v \sin i$ calculated from 16 different spectral lines at different wavelengths. Note that the velocities determined from the two C II lines at $6578 \AA$ and $6582 \AA$ are represented by one data point. The $\mathrm{H} \alpha$ lines were not used for the $v \sin i$ determination because they are intrinsically broadened and would not give accurate results.

and the synthetic line widths. This gives an accurate value of $24 \pm 6 \mathrm{~km} \mathrm{~s}^{-1}$. This value is an improvement and confirmation of the previously determined one of $\sim 30 \mathrm{~km} \mathrm{~s}^{-1}$ (Furenlid et al. 1987). In Fig. 5, we present the values obtained for $v \sin i$ and their calculated errors. A horizontal line gave the best fit for the data, implying that there were no systematic errors in the $v \sin i$ determination connected to the wavelengths where these velocities were measured. The first data point at $4479.86 \AA$ with a value of $13 \mathrm{~km} \mathrm{~s}^{-1}$ is given a lower weighting because of difficulties fitting the continuum in that region. Note that there are two data points with wavelengths longer than $6500 \AA$.

\subsection{Systemic velocity $\gamma$}

Variations observed in line profiles can be an indication of stellar pulsation. These profiles, however, also contain information relating to the movement of the system. Therefore, in order to isolate the features resulting from pulsation it is first necessary to calculate and subtract the systemic velocity from the spectra. This way, one obtains the spectra in the stellar rest frame and the pure stellar pulsation can be studied.

The systemic velocity $\gamma$ for $\mathrm{BW}$ Vul is derived at phase $\phi=0.54$ where the line profile is the narrowest and most symmetric, and the velocity field inside the line formation region is negligible. For the determination of the systemic velocity only unblended lines are used (two O II lines at $\lambda 4661.635 \AA$ and $\lambda$ 4676.231 $\AA$, and two Si III lines $\lambda 4567.840 \AA$ and $\lambda$ $4574.757 \AA$ ). The $\mathrm{H} \alpha$ line is not used because in hotter stars the Hydrogen lines are intrinsically broadened and should not be used for the determination of velocities. A mean value of $\gamma=-8.54 \pm 0.96 \mathrm{~km} \mathrm{~s}^{-1}$ is derived, which is in good agreement with the previously determined value of $\gamma=-9.2 \pm 1.7 \mathrm{~km} \mathrm{~s}^{-1}$ from Mathias et al. (1998). 
Table 5. Radial velocities.

\begin{tabular}{|c|c|c|c|c|c|c|c|}
\hline$\Phi$ & $\begin{array}{c}\mathrm{H} \alpha \\
6562 \AA\end{array}$ & $\begin{array}{c}\text { N II } \\
5710 \AA\end{array}$ & $\begin{array}{c}\text { C II } \\
6578 \AA\end{array}$ & $\Phi$ & $\begin{array}{c}\mathrm{He} \mathrm{I} \\
4387 \AA\end{array}$ & $\begin{array}{c}\text { Si III } \\
4567 \AA\end{array}$ & $\begin{array}{c}\text { O II } \\
4661 \AA\end{array}$ \\
\hline 0.01 & 14.14 & -0.73 & -3.51 & 0.06 & 29.99 & 28.88 & 22.83 \\
\hline 0.09 & 66.75 & 47.56 & 66.22 & 0.11 & 69.62 & 69.57 & 69.78 \\
\hline 0.13 & 115.94 & 67.51 & 70.78 & 0.17 & 70.99 & 70.88 & 72.35 \\
\hline 0.17 & 118.68 & 66.98 & 68.95 & 0.23 & 66.20 & 64.97 & 65.28 \\
\hline 0.21 & 117.31 & 62.79 & 64.40 & 0.29 & 56.64 & 55.13 & 55.63 \\
\hline 0.30 & 89.98 & 49.66 & 53.46 & 0.35 & 48.44 & 45.94 & 46.63 \\
\hline 0.34 & 73.58 & 44.41 & 43.89 & 0.43 & 33.41 & 30.85 & 31.19 \\
\hline 0.38 & 61.28 & 38.64 & 37.51 & 0.48 & 20.43 & 19.03 & 18.33 \\
\hline 0.42 & 47.62 & 32.86 & 29.30 & 0.54 & 6.76 & 5.25 & 5.47 \\
\hline 0.46 & 33.96 & 25.51 & 23.38 & 0.60 & -6.90 & -7.88 & -9.97 \\
\hline 0.52 & 14.14 & 11.34 & 9.71 & 0.66 & -24.66 & -24.94 & -26.05 \\
\hline 0.59 & -3.62 & -3.88 & -3.05 & 0.72 & -44.48 & -44.63 & -47.27 \\
\hline 0.64 & -22.07 & -15.96 & -15.36 & 0.78 & -64.29 & -66.94 & -66.56 \\
\hline 0.68 & -39.83 & -31.18 & -30.85 & 0.84 & -82.05 & -86.63 & -89.71 \\
\hline 0.72 & -61.01 & -46.93 & -44.98 & 0.87 & -62.24 & -56.44 & -72.35 \\
\hline 0.77 & -82.87 & -63.73 & -60.93 & 0.93 & 10.18 & 16.41 & 13.18 \\
\hline 0.81 & 107.47 & -77.90 & -75.97 & 1.00 & 7.45 & 7.88 & 6.11 \\
\hline 0.93 & -28.90 & 2.41 & 8.34 & & & & \\
\hline 0.96 & 17.56 & 7.14 & 6.52 & & & & \\
\hline 0.97 & 39.42 & 19.74 & -0.32 & & & & \\
\hline
\end{tabular}

\subsection{Radial velocity}

Radial velocity measurements of a star describe the Doppler shifts of the motions in the photosphere. In this study, the radial velocities are determined from spectral lines by calculating their central wavelengths and comparing them to the rest wavelengths using the formula for Doppler shift. The central wavelengths of the Doppler shifted lines are determined using IRAF's task splot where the option to fit a Gaussian profile was chosen. These measurements are obtained for six elements of the medium resolution data set, namely $\mathrm{H} \alpha$ at $6526.797 \AA$, Si III at $4567.84 \AA$, He I at $4387.929 \AA$, C II at $6578.053 \AA$, O II at $4661.635 \AA$ and N II at $5710.766 \AA$. All these lines are unblended and therefore suitable for the determination of radial velocities. The results are shown in Table 5. We estimate the errors on the radial velocity measurements to be $2.6 \mathrm{~km} \mathrm{~s}^{-1}$.

In Fig. 6, we show a comparison of the radial velocity curves of these lines. It is clear from this figure that the minima and maxima of different curves are reached at different times. Since these lines are formed in different regions of the photosphere, it appears that different layers of the atmosphere are moving out of phase with one another.

This phenomenon is called the Van Hoof effect and it was observed earlier between the $\mathrm{H}$ line and the metal lines (McNamara et al. 1955). In the following section we will study this effect for the six lines from which radial velocity measurements were obtained.

\subsubsection{Van Hoof effect}

The Van Hoof effect is defined as a phase lag of Hydrogen compared to metal lines (Van Hoof \& Struve 1953). This phase lag is the result of differential motion between the layers of an atmosphere in which Hydrogen and metal lines are formed. The
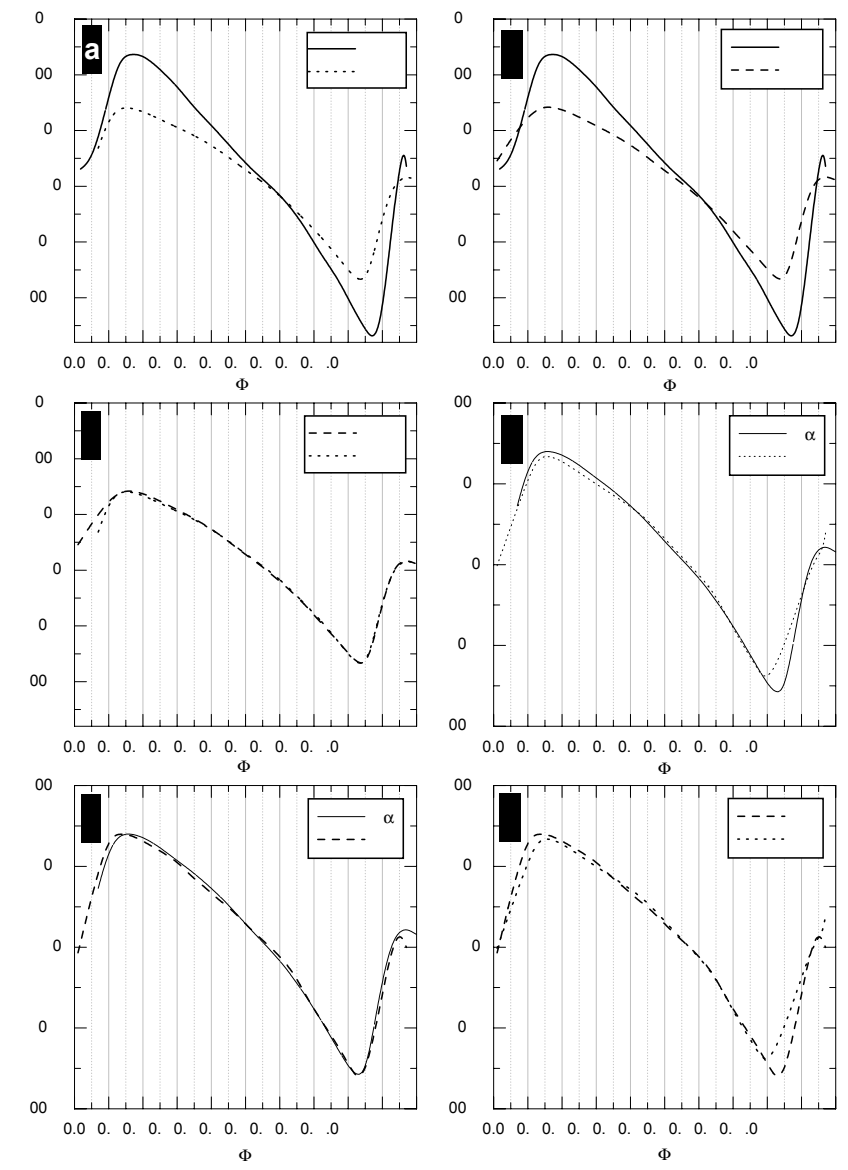

Fig. 6. Comparison of radial velocity curves of He I and Si III, He I and O II, O II and Si III, H $\alpha$ and N II, H $\alpha$ and C II, C II and N II. In panels a), b), d), and f), a phase lag between the radial velocities on theses lines is visible.

difference in formation depth originates in the higher ionization potential of Si III $(16.35 \mathrm{eV})$ compared to that of $\mathrm{H} \alpha(13.6 \mathrm{eV})$ as well as in the different excitation potentials of different lines. The phase lag is interpreted as the time that a pulsation wave needs to propagate through the atmosphere between the formation regions of these elements.

The Van Hoof effect in BW Vul was detected by McNamara et al. (1955) who found velocity differences of 35 to $40 \mathrm{~km} \mathrm{~s}^{-1}$ between the lines of Hydrogen $(\mathrm{H} \gamma$ and $\mathrm{H} \delta)$ and the average of all lines of all elements.

Mathias \& Gillet (1993) found that the Van Hoof effect is also present between the metal lines. They compared the radial velocities of the Si III line at $\lambda 4553 \AA$, the O II line at $\lambda 4591 \AA$, and the $\mathrm{H} \alpha$ line $\lambda 6563 \AA$. They note however, the same line doubling effects as were observed in this study (see Sect. 5), which make it difficult to obtain accurate values for the radial velocities.

As the present data spans a wavelength range of more than $3690 \AA$, it is possible to investigate for the first time whether the Van Hoof effect is present between many regions of the photosphere of BW Vul. A detailed comparison of the radial velocities of six unblended lines is performed: $\mathrm{H} \alpha$ at $6526.797 \AA$, Si III at $4567.84 \AA$, He I at $4387.929 \AA$, C II at $6578.053 \AA$, O II at $4661.635 \AA$, and N II at $5710.766 \AA$ (see Fig. 8). 

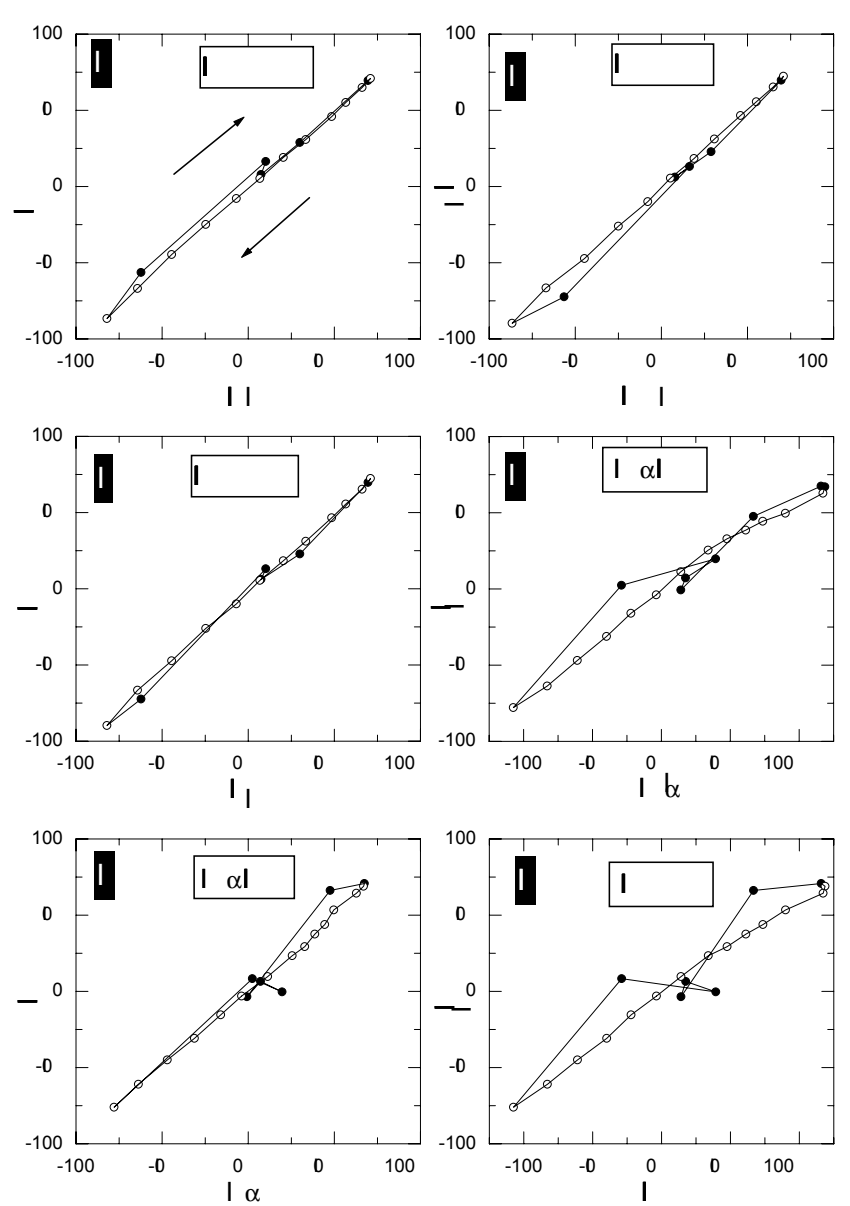

Fig. 7. Velocity-velocity curves of He I versus Si III, He I versus O II, Si III versus $\mathrm{O}$ II, $\mathrm{H} \alpha$ versus $\mathrm{N}$ II, $\mathrm{H} \alpha$ versus $\mathrm{C}$ II, and $\mathrm{N}$ II versus $\mathrm{C}$ II. The filled circles mark the ascending branch of radial velocity, the open circles mark the descending branch. The error bars are within the circles. The arrows indicate the way the curve is drawn.

The data were obtained on two different nights at different phases. Interpolation between these radial velocity values from different phases is not possible, because the change in velocity happens rapidly in BW Vul and the interpolated values would therefore be inaccurate. Consequently, only those spectral lines from observations made on the same night are compared to each other. The first night's data comprised He I, Si III, O II and the second night's data comprised $\mathrm{H} \alpha$, N II, C II. In Fig. 7 we show the radial velocity curves of He I and Si III (panel a), He I and O II (panel b), O II and Si III (panel c) as well as the curves for $\mathrm{H} \alpha$ and $\mathrm{N}$ II (panel d), $\mathrm{H} \alpha$ and $\mathrm{C}$ II (panel e) and N II and C II (panel f).

In panels $\mathrm{a}, \mathrm{b}, \mathrm{d}$, and $\mathrm{f}$, we see that the maxima and minima of the radial velocity curves are reached at slightly different times. This suggests that the Van Hoof effect is present between those lines, but these diagrams alone are not a stringent proof of the effect because the polynomial functions used to plot the radial velocity curves are not very accurate at times of minimum or maximum.

In order to verify the existence of the van Hoof effect in BW Vul, velocity-velocity diagrams are plotted for these six elements (see Fig. 7). These plots strongly indicate the occurrence of the Van Hoof effect in cases where loops form, and are made according to Mathias \& Gillet (1993). From the breadth of these loops, it is possible to directly infer the phase lag, and knowing the exact period $(P=0.20104444 \mathrm{~d}$, Sterken et al. 1993), one can also calculate the time the wave takes to propagate between the two layers of the atmosphere in which the lines are produced. One side of each loop is poorly defined in Fig. 7 as a result of the rapid changes in radial velocity, where the present data is not dense enough to form precisely definable loops. Only when these loops are well defined is it possible to use them to calculate the phase lag.

In the case of BW Vul, double loops form, each corresponding to one atmospheric acceleration, when the shock wave propagates outward. Because of these two loops, it is not easy to measure the amount of time that is needed for a wave to propagate between two layers. One reason is that no mathematical function, such as an elliptic equation, can be made to fit to the data. The second is that one would have to know the periods associated with one single loop in order to derive the propagation time of the wave. Nevertheless, a phase lag between the radial velocity curves derived from the different lines can be observed between $\mathrm{N}$ II and C II, and N II and $\mathrm{H} \alpha$, as well as between He I and Si III, and He I and O II (see Figs. 7 and 8). Note that the error bars for each point are contained within the symbol of each data point. There is a visible gap between the radial velocities of the above mentioned elements, and from this one can obtain an estimate of the propagation time between the layers. From Fig. 7, we estimated the phase shifts between N II and C II to be $9 \min 12.4 \mathrm{~s}$, for $\mathrm{N}$ II to $\mathrm{H} \alpha$ to be $9 \min 12.4 \mathrm{~s}$, and for He I to $\mathrm{Si}$ III to be $9 \mathrm{~min} 57.5 \mathrm{~s}$.

Although it is only possible to provide estimates of the phase lags from this analysis, our results verify that the Van Hoof effect is likely to be an overall property of BW Vul occurring between all lines.

\section{Conclusions}

We present the first ever abundance analysis of BW Vul showing a large over-abundance of helium for this star. It is possible that this results from the presence of a magnetic field which inhibits the mixing of elements in the stellar atmosphere. A magnetic field can also have an effect on the line profiles, which could explain some of the unusual spectral features observed in this star. Unfortunately it is not possible to confirm the presence of a magnetic field using the current data alone. This issue will be addressed in future work.

Radial velocity studies confirm the occurrence of the Van Hoof effect in BW Vul. While previous studies focussed primarily on line profile analyses of narrow spectral ranges containing only the $\mathrm{H} \alpha$ and Si III lines, the much broader wavelength range of the data used in this study allows for observation of the Van Hoof effect throughout many more layers in this star.

The rotational and systemic velocities are determined from a broader sample of lines than was previously possible. The resulting values match well and even improve those of earlier studies.

Details of the conclusions of this study are itemized below.

- We perform an abundance analysis and find that most elements have similar abundances to the Sun. Three 


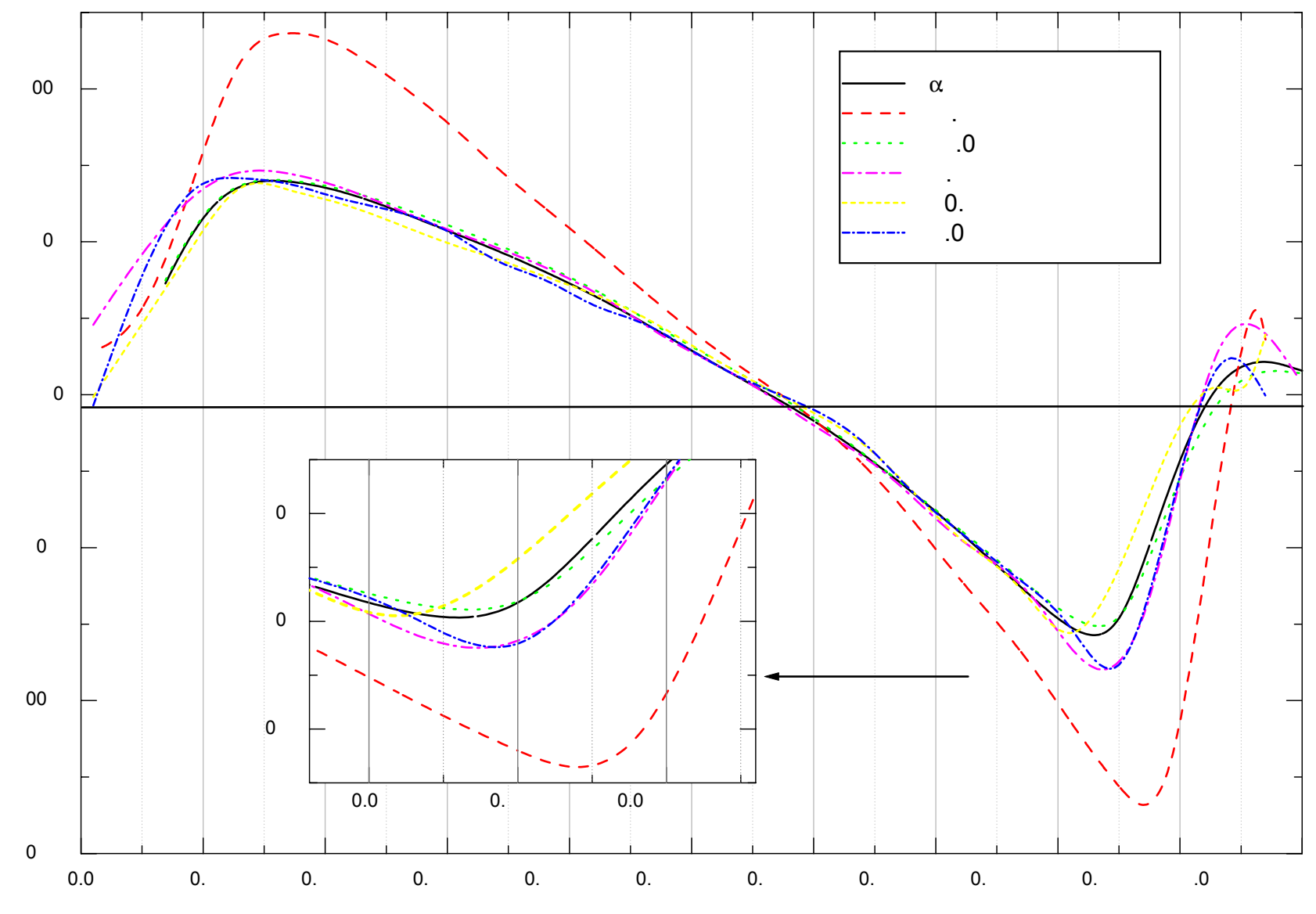

Fig. 8. Radial velocity curves of $\mathrm{H} \alpha$, He I, Si III, O II, N II, and C II.

remarkable exceptions are Neon, Silicon, and helium. Ne is over-abundant by $1.20 \pm 0.7 \mathrm{dex}, \mathrm{Si}$ is over-abundant by $1.00 \pm 0.48$ dex and He is over-abundant by $0.32 \pm 0.02$ dex. The He abundance might be connected to a magnetic field, which diminishes the mixing of elements. Such a magnetic field could be related to the strong linear behavior of BW Vul and will be investigated in more detail.

The Ne abundance is so far unexplained.

- We determine $v \sin i$ from 16 different lines and find good agreement between our result $\left(24 \pm 6 \mathrm{~km} \mathrm{~s}^{-1}\right)$ and the previously determined value of $\sim 30 \mathrm{~km} \mathrm{~s}^{-1}$ (Furenlid et al. 1987).

- The Van Hoof effect is clearly present in this star. We confirm the results of Mathias et al. (1998) who noted this effect between the Si III line at $4553 \AA$ and the $\mathrm{H} \alpha$ line at $6562 \AA$, and we demonstrate that this effect is occurring for 6 different layers of the atmosphere corresponding to six different elements, and thus to six different excitation and ionization energies. We show that the Van Hoof effect is operating throughout most of the atmosphere.

- We analyze the radial velocity variations in the lines of six elements in BW Vul, and find a maximum peak-topeak amplitude of more than $220 \mathrm{~km} \mathrm{~s}^{-1}$ for the spectral line identified with He I $\lambda 4387.929 \AA$. This is significantly higher than the second highest peak-to-peak radial velocity amplitude, which was derived from O II $\lambda$ 4661.635 $\AA$ and reaches about $150 \mathrm{~km} \mathrm{~s}^{-1}$. The amplitudes of the other lines under study are all around $150 \mathrm{~km} \mathrm{~s}^{-1}$ as well.
It should be noted that BW Vul is a special case among the $\beta$ Cephei class, exhibiting many peculiar properties. Therefore caution should be used when trying to apply the results of any study of BW Vul to the other $\beta$ Cepheids.

\section{Future work}

In the future, we plan to obtain and analyze polarimetric data to investigate whether BW Vul has a magnetic field that contributes to its extraordinary behavior. We also aim to acquire high temporal and spectral resolution data over one whole pulsation cycle. These data will provide us with accurate radial velocity measurements for a larger sample of elements and will allow us to study the kinematics of the atmosphere of BW Vul, and to develop a 3-D velocity field model.

In combination, these studies will allow us to develop a more comprehensive view of BW Vul, and may help us to better understand the physical processes occurring within its atmosphere.

Acknowledgements. We thank Theresa Lueftinger and Peter Mittermayer from the Institute for Astronomy, Vienna, for support with spectroscopy and Prof. Nikolai Piskunov from the Dept. of Astronomy and Space Physics, Uppsala, Sweden for his programs and the constructive discussions.

This work was originally defined by Ingemar Furenlid who sadly passed away in 1994. 


\section{References}

Aerts, C., De Pauw, M., \& Waelkens, C. 1992, A\&A, 266, 294 Aerts, C., Mathias, P., Van Hoolst, T., et al. 1995, A\&A, 301, 781

Balona, L. A. 1984, MNRAS, 211, 973

Dziembowski, W. \& Pamyatnykh, A. 1993, MNRAS, 262, 204

Furenlid, I., Young, A., Meylan, T., Haag, C., \& Crinklaw, G. 1987, ApJ, 319, 264

Hauck, B. \& Mermilliod, M. 1998, A\&AS, 129, 431

Heynderickx, D., Waelkens, C., \& Smeyers, P. 1994, A\&AS, 105, 447

Iglesias, C. A., \& Rogers, F. J. 1991, ApJ, 371, L73

Ilyin, I. 2000, Ph.D. Thesis, University of Oulu, Finnland

Kupka, F., \& Lueftinger, T. 2001, in Proc. of the COROT/SWG Milestone 2000 meeting, ed. E. Michel \& A. Hui-Bon-Hoa

Kupka, F., Piskunov, N. E., Ryabchikova, T. A., Stempels, H. C., \& Weiss, W. W. 1999, A\&AS, 138, 119

Kurucz, R. L. 1992, in The Stellar Populations of Galaxies, IAU Symp., 149, 225
Mathias, P., \& Gillet, D. 1993, A\&A, 278, 511

Mathias, P., Gillet, D., Fokin, A. B., \& Cambon, T. 1998, A\&A, 339, 525

McNamara, D. H., Struve, O., \& Bertiau, F. C. 1955, ApJ, 121, 326

Moskalik, P., \& Dziembowski, W. A. 1992, A\&A, 256, L5

Pamyatnykh, A. A. 1999, Acta Astron., 49, 119

Pigulski, A. 1993, A\&A, 274, 269

Piskunov, N. E. 1992, in Stellar Magnetisms, ed. Y. V. Glagolevskij, \& I. I. Romanyuk

Piskunov, N. E., Kupka, F., Ryabchikova, T. A., Weiss, W. W., \& Jeffrey, C. 1995, A\&AS, 112, 525

Ryabchikova, T. A., Piskunov, N. E., Stempels, H., Kupka, F., \& Weiss, W. W. 1998, in Proc. of the 6th Int. Colloq. on Atomic Spectra and Oscillator Strengths, 162

Sterken, C., Pigulski, A., \& Liu, Z. 1993, A\&AS, 98, 383

Tuominen, I., Ilyin, I., \& Petrov, P. 1999, in Astrophysics with the NOT, ed. H. Karttunen, \& V. Piirola, 47

Van Hoof, A. \& Struve, O. 1953, PASP, 65, 158 